\title{
INCOMPLETE NORMED ALGEBRAS
}

\author{
BY BERTRAM YOOD ${ }^{1}$ \\ Communicated July 1, 1971
}

The Jacobson theory for rings is not well adapted to the study of a topological ring $R$ because the basic items of that theory such as the primitive ideals, the modular maximal right ideals and the radical need not be closed in $R$. In special cases, such as Banach algebras [7] or more generally $Q$-rings [3], all these items are closed while in some other cases such as locally compact rings [5] one has the radical closed but not necessarily the primitive ideals or the modular maximal right ideals.

We approach the study of ideal theory for $R$ by using only closed onesided or two-sided ideals (ideals are two-sided unless otherwise specified). A number of approaches are outlined below each providing useful conclusions. The results obtained are sharpest in case $R$ has the additional structure of being an (incomplete) normed algebra. Detailed proofs will appear elsewhere.

Examples show [10] that a right ideal in $R$ can be a maximal-closed modular right ideal without being a maximal modular right ideal even for normed algebras. Call an ideal $K$ topologically primitive if it has the form $K=(M: R)=\{x \in R: R x \subset M\}$ and denote by the topological radical, top $\operatorname{rad} R$, the intersection of all topologically primitive ideals. A theory of topologically primitive ideals is developed. In some ways it differs from the usual theory for primitive ideals. For example, if $K$ is a topologically primitive ideal in $R$ and $I$ is an ideal in $R$, then $K \cap I$ need not be a topologically primitive ideal in $I$. Here we focus attention on the following question. Let $\mathfrak{P}_{r}\left(\mathfrak{P}_{l}\right)$ be the intersection of the maximal-closed modular right (left) ideals. Is $\mathfrak{P}_{r}=\mathfrak{P}_{l}=$ top $\operatorname{rad} R$ (in analogy with primitive ideal theory)?

We find the answer to be affirmative for certain classes of normed algebras. A first rather easy case is for a normed algebra which is a dense ideal in a Banach algebra.

For a complex normed algebra $B$ with involution $x \rightarrow x^{*}$ let $P$ denote the closure in the set of all selfadjoint elements of the set of all finite sums of elements of the form $x^{*} x$ ( $P$ is the "positive cone" of $B$ ). In these terms we have the following result.

AMS 1970 subject classifications. Primary 46H10; Secondary 46K05.

Key words and phrases. Topological rings, normed algebras, Banach algebras.

${ }^{1}$ Partial support by NSF (GP-20226) is gratefully acknowledged, and NSF GP-28250. 
THEOREM 1. Let $B$ a complex normed algebra with an involution. If $B$ is semisimple and $P \cap(-P)=(0)$, then $\mathfrak{P}_{r}=\mathfrak{P}_{l}=$ top $\operatorname{rad} B=(0)$.

As a quite special case of Theorem 1 , any dense $*$-subalgebra $B$ of a complex Banach algebra $B_{0}$ with involution and faithful $*$-representation satisfies these conclusions. In particular $B$ can be any dense $*$-subalgebra of a $B^{*}$-algebra or of the group algebra of any locally compact group.

THEOREM 2. Let $B$ be a dense subalgebra of a Banach algebra $B_{0}$ which has the property that any closed one-sided ideal $\neq(0)$ contains a nonzero idempotent. Then $\mathfrak{P}_{r}=\mathfrak{P}_{l}=$ top $\operatorname{rad} B=(0)$.

In particular this holds if $B$ is a dense subalgebra of a semisimple annihilator Banach algebra [1] and so examples abound.

Next let $\mathfrak{D}_{r}\left(\mathfrak{D}_{l}\right)$ be the intersection of the closed modular maximal right (left) ideals. With each closed modular maximal right ideal $M$ we consider the associated closed primitive ideal $(M: R)$. It is known $[6, \mathrm{p} .36]$ that $\mathfrak{D}_{r}$ is the intersection of these associated primitive ideals and hence is an ideal. An open question is whether or not $\mathfrak{D}_{r}=\mathfrak{D}_{l}$ must hold. It does if $R$ is locally compact [5].

THEOREM 3. Let $R$ be a dense *-subring of a topological ring $R_{0}$ with an involution. Suppose that, in $R_{0}$, every closed ideal is a*-ideal. Then, for the ring $R, \mathfrak{D}_{r}=\mathfrak{D}_{l}$.

From this it follows that $\mathfrak{D}_{r}=\mathfrak{D}_{l}$ for a dense $*$-subalgebra of a $B^{*}$-algebra or of semisimple dual Banach algebra with involution (see [4]). The type of argument used in the proof of Theorem 3 also shows that $\mathfrak{D}_{r}=\mathfrak{D}_{l}$ for a Hilbert algebra (for this notion see [2]).

Next let $A$ be a semiprime topological ring (no nonzero nilpotent onesided ideals). Proper closed prime ideals arise naturally in the study of $A$ in the following way. For an ideal $I$ in $A$, its left annihilator $\{x \in A: x I=(0)\}$ coincides with its right annihilator $\{x \in A: I x=(0)\}$; call this set $I^{a}$. Let $\mathfrak{N}(A)$ denote the set of ideals $I$ for which $I^{a} \neq(0)$ and consider $\mathfrak{N}(A)$ as a partially ordered set under set inclusion. Its maximal elements are precisely the proper closed prime ideals $I$ in $A$ for which $I^{a} \neq(0)$.

Call $A$ a generalized annihilator ring (generalized dual ring) if $I^{a} \neq(0)$ $\left(I^{a a}=I\right)$ for all closed ideals $I \neq A$. Special cases are the semisimple annihilator rings of $[\mathbf{1}]$ (dual rings of [4]). A theory of these rings is developed. The ideas are interrelated. If $A$ is a generalized annihilator ring, every closed ideal has this property if and only if $A$ is a generalized dual ring. Among other results we obtain the following decomposition theorems.

THEOREM 4. $A$ is the direct topological sum of its minimal closed ideals if and only if it is a generalized annihilator ring and the intersection of its closed prime ideals is $(0)$. 
For a normed algebra $B$ we can say more.

THEOREM 5. Let $B$ be a normed algebra whose completion is semisimple. Then $B$ is the direct topological sum of its minimal closed ideals if and only if $B$ is a generalized annihilator normed algebra.

This provides an improvement on standard decomposition theorems such as in [1], [8] and [9] for Banach algebras.

\section{REFERENCES}

1. F. F. Bonsall and A. W. Goldie, Annihilator algebras, Proc. London Math. Soc. (3) 4 (1954), 154-167. MR 15, 881.

2. J. Dixmier, Les algèbres d'opérateurs dans l'espace Hilbertien (algèbres de von Neumann), Gauthier-Villars, Paris, 1969.

3. I. Kaplansky, Topological rings, Amer. J. Math. 69 (1947), 153-183. MR 8, 434.

4. - Dual rings, Ann. of Math. (2) 49 (1948), 689-701. MR 10, 7.

5. , Locally compact rings. II, Amer. J. Math. 73 (1951), 20-24. MR 12, 584.

6. E. A. Michael, Locally multiplicatively-convex topological algebras, Mem. Amer. Math. Soc. No. 11 (1952), 79 pp. MR 14, 482.

7. C. E. Rickart, General theory of Banach algebras, The University Series in Higher Math., Van Nostrand, Princeton, N.J., 1960. MR $22 \# 5903$.

8. M. F. Smiley, Right annihilator algebras, Proc. Amer. Math. Soc. 6 (1955), 698-701. MR 17, 386.

9. B. J. Tomiuk, Structure theory of complemented Banach algebras, Canad. J. Math. 14 (1962), 651-659. MR 26 \#626.

10. B. Yood, Ideals in topological rings, Canad. J. Math. 16 (1964), 28-45. MR 28 \# 1505.

Department of Mathematics, University of Oregon, Eugene, Oregon 97403 